\title{
Analysis of Ebola Epidemic Outbreaks in West Africa
}

\author{
Talawar A. S. and Pujar H. S. \\ Department Statistics, Karnatak University, Dharwad, India
}

\begin{abstract}
In the present paper, we used a SEIR model developed by Astacio, et al., (1996) for the 2014 outbreak of Ebola in Guinea, Liberia, and Sierra Leone. The data we have used for this outbreak is obtained from the Center for Disease Control (CDC: https://www.cdc.gov). We estimated the per capita growth rate of the disease and then, $\beta$ and $R_{0}$ are calculated for each of these countries. The estimates of the initial rate of growth $r$ for the Guinea, Liberia, and Sierra Leone for the 2014 outbreak (25-3-2014 to 30-3-2016) are 0.042 per week, 0.105 per week and 0.072 per week respectively. We have carried out analysis of the optimal level of effort to control the spread of ebola considering two control measures such as, vaccinating susceptible $\left(u_{1}\right)$ and quarantine of the symptomatic infective individuals $\left(u_{2}\right)$. Numerical illustration of the model is given for Guinea, Liberia, and Sierra Leone.
\end{abstract}

\section{Introduction}

Ebola Virus Disease (EVD) spread to humans and among humans through contact with the blood, secretions, organs, or other bodily fluids of those infected. It is not transmitted through the air. Individuals who are not symptomatic are not contagious. The largest and first regional outbreak of EVD has been occurred in West Africa during December 2013, but it was not recognised until March 2014. On March 22, 2014, the World Health Organization (WHO) announced that 49 people had contracted EVD in Guinea, West Africa and 29 of them had died. The World Health Organization (WHO) declared this EVD epidemic a Public Health Emergency of International concern on 8 August 2014. A total of 3,707 cases (including 2,106 confirmed, 1,003 probable and 598 suspected cases) and 1,848 deaths (concerning 1,050 confirmed and 557 probable cases, as well as 241 suspected cases and deaths) have been reported in Guinea, Sierra Leone, Liberia, Nigeria, and Senegal as of 31 August 2014. As of mid-August, the virus had spread to Sierra Leone, Liberia, and Nigeria, infecting more than 2,000 people and killing over half of them (Figure 1).

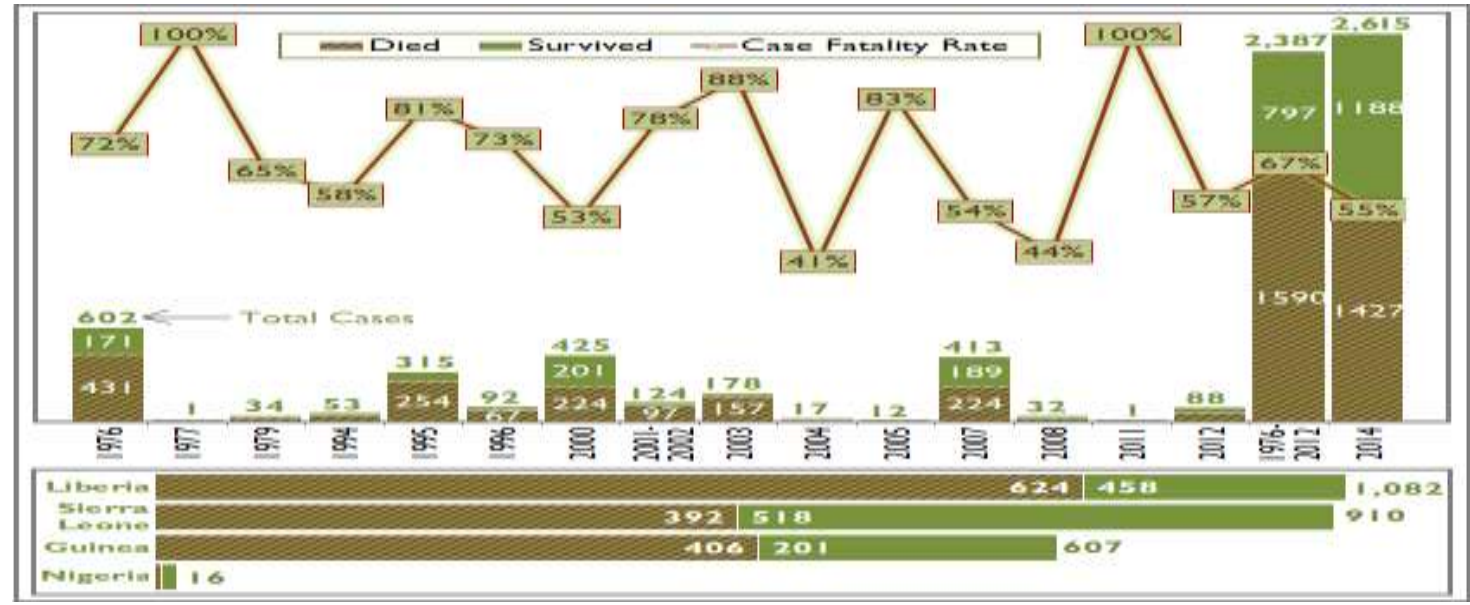

Figure 1: Ebola Outbreaks: 1976-2014, as Reported on August 22, 2014: Source: CRS (2014).

Prior to the current outbreak in Guinea, Liberia, and Sierra Leone, EVD outbreaks were concentrated in the DRC, Gabon, the Sudans, and Uganda (Figure 2). A major factor in the rapid spread of Ebola among the affected countries is weak health system capacity. Guinea, Liberia, and Sierra Leone are among the poorest countries in the world. The infrastructure, including the health systems, of these countries has been decimated by years of conflict and neglect. Despite improved control measures, Ebola remains a serious public health risk in African regions where recurrent outbreaks have been observed since the initial epidemic in 1976 


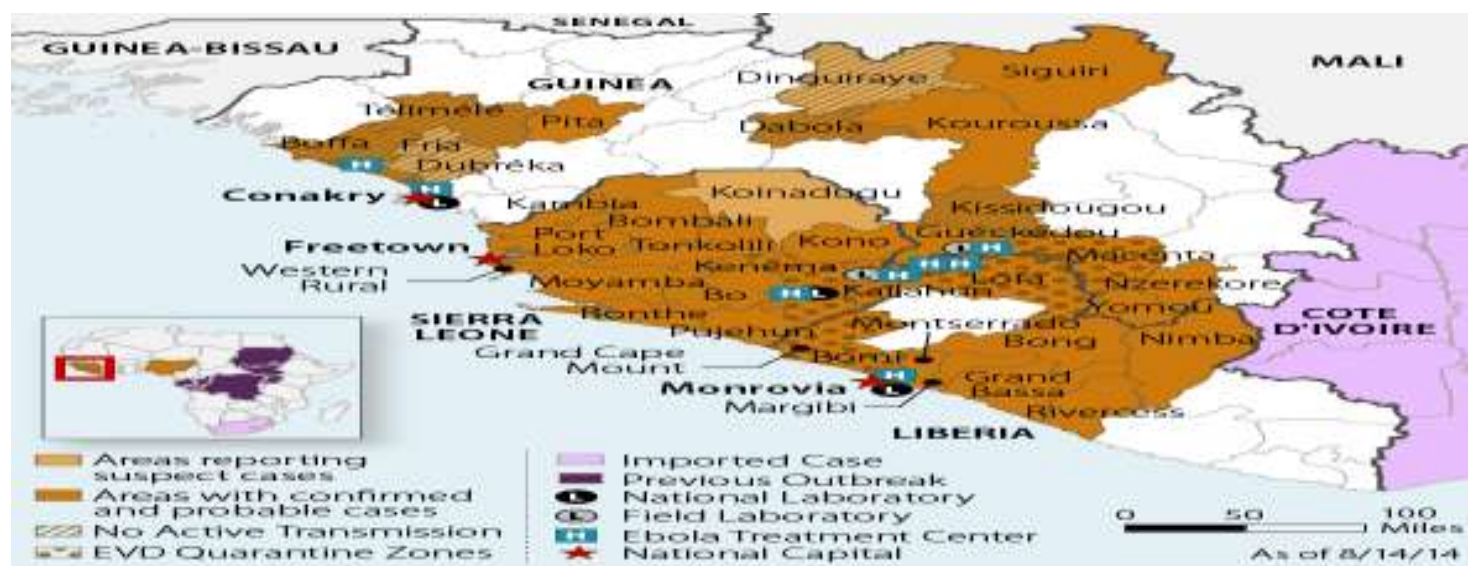

Figure 2: Map of Current and Past Ebola Outbreaks: Source: Adapted by CRS from the Centers of Disease Control and Prevention (CDC) at resources/distribution-map-guinea-outbreak.html

The World Health Organization (WHO) is responsible for determining when a country will be declared free of Ebola virus transmission. On December 29, 2015, WHO declared Guinea free of Ebola virus transmission. On November 7, 2015, WHO declared Sierra Leone free of Ebola virus transmission and two new confirmed cases were reported in January 2016. On March 17, 2016, WHO declared the end of the flare-up after 42 days had passed since the last Ebola patient tested negative. WHO first declared Liberia free of Ebola virus transmission on May 9, 2015. The country subsequently experienced a cluster of six Ebola cases in June 2015 and was declared free of transmission again on September 3, 2015. A second cluster of three cases was reported in November 2015, and WHO declared the country free of transmission for the third time on January 14, 2016 (Figure 3, 4 and 5).

Table 1: Total number of cases, laboratory confirmed cases and deaths of 2014 Ebola outbreak in West Africa.

\begin{tabular}{|c|c|c|c|}
\hline Country & $\begin{array}{c}\text { Total Cases (Suspected, } \\
\text { Probable, and Confirmed) }\end{array}$ & $\begin{array}{c}\text { Laboratory-Confirmed } \\
\text { Cases }\end{array}$ & Total Deaths \\
\hline Guinea & 3814 & $3358(88.04)$ & $2544(66.70)$ \\
\hline Liberia & 10678 & $3163(29.62)$ & $4810(45.05)$ \\
\hline Sierra Leone & 14124 & $8706(61.64)$ & $3956(28.01)$ \\
\hline Total & 28616 & $15227(53.21)$ & $11310(39.52)$ \\
\hline
\end{tabular}

From the Table 1, it is noticed that more than $88 \%$ are confirmed cases out of 3814 suspected cases for Guinea, nearly $30 \%$ cases found confirmed out of total 14124 suspected cases for Liberia and about $62 \%$ cases are found confirmed for Sierra Leone. About $67 \%, 45 \%$ and $28 \%$ of deaths have respectively occurred due to ebola in Guinea, Liberia and Sierra Leone.

\section{Modelling of EVD}

Mathematical/Statistical modelling has emerged as an important tool for understanding of the dynamics of the spread of infectious diseases. The theoretical framework most commonly used is based on the division of the human host population into three categories containing susceptible, infected and recovered individuals (S-IR) and into four categories containing susceptible, infected but not yet infectious (exposed), infectious, and recovered individuals (S-E-I-R). Chowell et al. (2004a) estimated the number of secondary cases generating by an index case in the absence of control interventions using epidemic modelling and data from two welldocumented Ebola outbreaks (Congo 1995 and Uganda 2000). Chowell et al. (2004b) considered only a part of the available data on this outbreak for estimating the parameters of a set of deterministic SEIR differential equations with a time-varying transmission rate to allow for the control intervention. Their estimation approach is based on simulating the solutions to the deterministic SEIR equations and identifying the parameter values that minimize the sum of squared errors between the observed and simulated cumulative number of cases. The optimization process was started from 10 different initial parameter values and the reported parameter estimate is the one that resulted in the smallest sum of squares of error. Lekone and Finkenstädt (2006) developed a stochastic discrete-time susceptible-exposed-infectious-recovered (SEIR) model for infectious diseases with the aim of estimating parameters from daily incidence and mortality time series for an outbreak of Ebola in the Democratic Republic of Congo in 1995. In order to investigate the transmission mechanism of the infectious individual with Ebola virus, Li et al., (2015) established an SEIT (susceptible, exposed in the latent period, infectious, and treated/recovery) epidemic model and with suitable parameter values, they obtained the estimated value of the basic reproduction number and analyzed the sensitivity and uncertainty property by partial rank correlation coefficients. 


\section{The Model}

In the present paper, we used a SEIR model developed by Astacio, et al., (1996) for the 2014 outbreak of Ebola in Guinea, Liberia, and Sierra Leone. The data we have used for this outbreak is obtained from the Center for Disease Control (CDC: https://www.cdc.gov). In this model, we differentiate between the incubation period and the infectious period of the disease. As before, the number of susceptible individuals at time $t$ will be denoted as $S(t)$. We refer to the incubation period of the disease as the latent stage. Therefore the number of latent individuals at time $t$ will be denoted by $E(t)$. Individuals that are infected with the disease and are symptomatic of Ebola will be classified as infectious individuals. The number of infectious individuals at time $t$ will be denoted by $I(t)$. Similarly, the number of dead /recovered individuals at time $\mathrm{t}$ will be denoted by $R(t)$. We consider a constant population during the outbreak (without demographic factors such as birth rate, natural death rate and migration); i.e., the total population at time t will be denoted by $N$, where $N=S(t)+E(t)+I(t)+$ $R(t)$. Thus the $S-E-I-R$ model is expressed as the systems of differential equations

$\frac{d S}{d t}=-\beta S(I+q E) / N$

$\frac{d E}{d t}=\beta S(I+q E) / N-\delta E$

$\frac{d I}{d t}=\delta E-\gamma I$

$\frac{d R}{d t}=\gamma I$

In (1) the term $\beta S(I+q E) / N$ takes into account of the number of people infected due to direct contact with an infected individual and the number of people infected due to direct contact with a latent individual. The parameter $q(0 \leq q \leq 1)$ is a weight factor added to the model since it is known that a susceptible individual has a higher chance of getting infected from an infectious individual than from a latent individual (Astacio, et al., 1996)

As we see in (1), the individuals in the incubation period eventually show the symptoms of the disease, and pass on to the infectious stage. The quantity is denoted by $\delta E$, where $\delta$ is the per-capita infectious rate. Then $1 / \delta$ becomes the mean time for a latent individual to become infectious. The rate at which infectious individual recover/die is denoted by $\gamma I$, where $\gamma$ is the per-capita death rate and $1 / \gamma$ becomes the mean time it takes an individual to recover/die once latent individual has entered the infectious stage. Figure 6 gives the number of people who became infected and dead each week during the outbreak in West Africa.

The important number, that is the basic reproductive number or basic reproductive rate $\left(R_{0}\right)$ of Ebola, which is very useful for the effects of public health measures, needs to be computed using the 2014 Ebola outbreak in West Africa. This number tells us how fast the disease will spread at the beginning of the epidemic and how to carry out the interventions to protect population from the disease. To calculate the value for $R_{0}$, we need to evaluate the Jacobian matrix of the system of differential equations (1) at the disease-free equilibrium (DFE) point and the DFE point is $(S, E, I, \mathrm{R})=(N, 0,0,0)$. We reduce the four- dimensional system to a threedimensional system by neglecting the fourth equation of (1), because fourth equation is not appeared in any of the first three equations of (1). Once the Jacobian is evaluated at this point, the determinant and the trace must both be greater than zero to insure that the disease-free state is an unstable fixed point. Therefore $R_{0}$ for (1) is obtained as

$$
R_{0}=\left(\frac{\beta}{\gamma}\right)(1+q \gamma / \delta)
$$

If all the parameter values are estimated, the system of differential equations can be solved easily.

\section{Sensitivity and Uncertainty analysis on $R_{0}$}

Chowell et al. (2004a) used a log-normal distribution for the incubation period of Ebola to study uncertainty analysis. Log- normal distribution parameters are set from empirical observations (mean incubation period is 6.3 and the $95 \%$ quantile is 21 days (Breman et al., 1977). The infectious period is assumed to be uniformly distributed in the range (3.5-10.7) days (Piot et al., 1977). A formula for the basic reproductive number $R_{0}$ that depends on the initial per-capita rate of growth $\mathrm{r}$ in the number of cases (Figures 3-5), the incubation period $1 / \delta$ and the infectious period $1 / \gamma$ can be obtained by linearizing the equations $\frac{d E}{d t}$ and $\frac{d I}{d t}$ of system (1) around the DFE point with $\mathrm{S}=\mathrm{N}$. The corresponding Jacobian matrix is given by

$$
\mathbf{J}=\left[\begin{array}{cc}
\beta q-\delta & \beta \\
\delta & -\gamma
\end{array}\right]
$$

And the characteristic equation is given by

$$
r^{2}-(\beta q-\delta-\gamma) r-(\gamma(\beta q-\delta)+\beta \delta)=0
$$

By solving the characteristic equation for $\beta$ in terms of $\mathrm{r}, \delta, \gamma$ and $\mathrm{q}$, we get 
$\beta=\frac{r^{2}+(\delta+\gamma) r+\gamma \delta}{(q r+\delta-q \gamma)}$

Using the expression for $R_{0}$ and substituting for $\beta$, we obtain

$R_{0}=\frac{\left(r^{2}+(\delta+\gamma) r+\gamma \delta\right)(q \gamma+\delta)}{\gamma \delta(q r+\delta-q \gamma)}$

From the Figures 3-5, we estimate the per capita growth rate of the disease and then, we estimate $\beta$. There fore $R_{0}$ is calculated for each of these countries. The estimates of the initial rate of growth $\mathrm{r}$ for the Guinea, Liberia, and Sierra Leone for the 2014 outbreak (25-3-2014 to 30-3-2016) are 0.042 per week, 0.105 per week and 0.072 per week respectively. Which are obtained from the time series data $x(t)$, the cumulative total of the number of cases and assuming exponential growth $\left(x(t) \propto e^{r t}\right)$. Figures 7, 8 and 9 give the observed and fitted values. The following Table 2 and 3 give the parameters values for the Guinea, Liberia, and Sierra Leone 2014 epidemic.

Table 2 and 3: Estimated values of $\beta, \delta, \gamma, \mathrm{q}$ and $R_{0}$

\begin{tabular}{|c|c|c|c|c|c|}
\hline & $\beta$ & Incubation period $(1 / \delta)^{*}$ & Infectious period $(1 / \gamma)$ & $q$ & $R_{0}$ \\
\hline Guinea & $\begin{array}{c}0.264 \\
(0.195)\end{array}$ & $\begin{array}{c}0.188 \\
\text { (5.32 days) }\end{array}$ & $\begin{array}{c}0.16 \\
\text { (6.25 days) }\end{array}$ & 0.15 & $\begin{array}{c}1.923 \\
(1.376)\end{array}$ \\
\hline Liberia & $\begin{array}{c}0.380 \\
(0.217) \\
\end{array}$ & $\begin{array}{c}0.251 \\
\text { (3.98 days) }\end{array}$ & $\begin{array}{c}0.16 \\
\text { (6.25 days) }\end{array}$ & 0.25 & $\begin{array}{c}2.881 \\
(1.571)\end{array}$ \\
\hline Sierra Leone & $\begin{array}{c}0.377 \\
(0.293)^{*}\end{array}$ & $\begin{array}{c}0.223 \\
\text { (4.48 days) }\end{array}$ & $\begin{array}{c}0.21 \\
\text { (4.76 days) }\end{array}$ & 0.24 & $\begin{array}{c}2.558 \\
(1.713)^{*}\end{array}$ \\
\hline
\end{tabular}

- Figures in parenthesis give the values for per day

\begin{tabular}{|c|c|c|c|c|}
\hline & $\beta$ & Incubation period $(1 / \delta)^{*}$ & Infectious period $(1 / \gamma)$ & $R_{0}$ \\
\hline Guinea & $\begin{array}{c}0.264 \\
(0.171)\end{array}$ & $\begin{array}{c}0.188 \\
\text { (5.32 days) }\end{array}$ & $\begin{array}{c}0.16 \\
\text { (6.25 days) }\end{array}$ & $1.923(1.071)$ \\
\hline Liberia & $\begin{array}{c}0.380 \\
(0.185)\end{array}$ & $\begin{array}{c}0.251 \\
\text { (3.98 days) }\end{array}$ & $\begin{array}{c}0.16 \\
\text { (6.25 days) }\end{array}$ & $\begin{array}{c}2.881 \\
(1.159)\end{array}$ \\
\hline Sierra Leone & $\begin{array}{c}0.377 \\
(0.230)^{*} \\
\end{array}$ & $\begin{array}{c}0.223 \\
\text { (4.48 days) } \\
\end{array}$ & $\begin{array}{c}0.21 \\
\text { (4.76 days) } \\
\end{array}$ & $\begin{array}{c}2.558 \\
(1.097)^{*} \\
\end{array}$ \\
\hline
\end{tabular}

- Figures in parenthesis give the values per day when $\boldsymbol{q}=\mathbf{0}$

The calculated $R_{0}$ values ranged from 2.6 to 8.6 for the Yambuku, Zaire outbreak in 1976; meanwhile the range for $R_{0}$ for the 1995 epidemic in Kikwit, Zaire was slightly lower: $1.57 \leq R_{0} \leq 5.03$ (Astecia, et al., 1996) and for Congo epidemic it was between 1.66 and 2.28 (Chowell et al., 2004a). The calculated $R_{0}$ values for Guinea, Liberia and Sierra Lione are respectively given by the range 1.55-7.68, 1.47-4.55 and 1.43-11.23. The number of deaths can be minimized by altering the environment; i.e., lowering $\beta$ (the effective contact rate). This can be done by implementing the quarantine application.

\section{Limitations of the model}

The model which we have considered here is used a constant effective contact rate, $\beta$. This is probably not the best case for $\beta$ since the probability of contracting the Ebola virus varies as the disease becomes more widespread. People are more careful with whom they have contact, and thus the number of contacts decreases as time elapses or as the number of infected increases. Therefore, it makes sense to have $\beta$ as the time dependent. This limitation is achieved by Chowell et al., (2004b) and, Lekone and Fenkenstädt (2006). Another idea for improving the model is to consider quarantine application. When infected people are isolated, the number of contacts that can transmit the disease automatically decreases. Here we have used arbitrary values for $\mathrm{q}$, so that the model best fits the available data. But it is clear that individuals showing symptoms of the Ebola disease are more infectious than latent individuals who show no symptoms. Therefore, a better value for $q$ would be more accurate in predicting the dynamics of a future Ebola outbreak (Astacio, et al., 1996). The World Health Organization (WHO) provided data on the 2014 Ebola outbreak in Liberia, Sierra Leone, and Guinea. In the present paper we have divided the entire period of outbreak into four phases. The period for phase I from 25-32014 to 22-8-2014, for phase II from 28-8-2014 to 21-11-2014, for phase III from 26-11-2014 to 25-2-2015 and for phase III from 2-3-2015 to 30-3-2016. We have estimated $\beta$ and $R_{0}$ at various exponential growth rates using ebola outbreak data of Liberia, Liberia and Sierra Leone for different phases (Figures 10, 11 and 12). 
We modified the model (1) including two parameters: vaccination and quarantine. The modifications portray the strategies used by countries to eliminate the disease. Optimal control is a powerful optimization technique used to derive the best control strategies. A set of differential equations are used with specific control rates that minimize an objective functional, which include all the variables that will be minimized. Pontryagin's maximum principle validates the existence of an optimal control (Lenhart, and Workman, 2007). Optimal control is applied to the SEIR model. The two control measures are use of vaccination and quarantine application. Quarantine is currently a procedure that is heavily utilized in order to maintain infected individuals. Both of these control measures are contrasted and analyzed over time.

\section{SEIR Model with vaccination and quarantine}

We formulate a four component SEIR (Susceptible-Exposed-Infected-Recovered) model (1) that contains both vaccination and quarantine as ways to decrease the susceptible and infected.

$\frac{d S}{d t}=-\frac{\beta S(I+q E)}{N}-u_{1} S$

$\frac{d E}{d t}=\frac{\beta S(I+q E)}{N}-\delta E$

$\frac{d I}{d t}=\delta E-\left(u_{2}+\gamma\right) I$

$\frac{d R}{d t}=\gamma I+u_{1} S+u_{2} I$

The interaction term $\frac{\beta S(I+q E)}{N}$ is crucial to the model. A certain amount of interactions, dependent on the transmission rate $\beta$, between a susceptible and an infected will result in the susceptible contracting a latent form of Ebola. Thus, that susceptible will move into the exposed class. However, a certain amount of susceptibles will be vaccinated $u_{1} S$ and move straight into the recovered class without becoming infected. The exposed compartment decreases at some rate $\delta$, because latent form of the virus becomes infectious. Similarly, some of the infected $\gamma I$ will recover without treatment and move into the recovered class. One equilibrium exists for this model, the disease free equilibrium (DFE). The DFE, where there are no infected or $\mathrm{I}=0$, is $P_{0}=(\mathrm{N}, 0,0,0)$. Since there is no birth rate in this system, it is a trivial equilibrium.

\section{Application of Optimal Control Theory}

For analysis of the optimal level of effort required to control the spread of ebola, two control measures are taken into consideration, vaccinating susceptible $\left(u_{1}\right)$ and quarantine of the symptomatic infective individual $\left(u_{2}\right)$. We will consider the finite-dimensional optimal control problem. In order to formulate an optimal control problem, a state and objective functional are required. Consider the following objective functional $\mathrm{J}(\mathrm{u})$ subject to some differential equation (state)

$J(u)=\int_{t_{0}}^{t_{1}} f(t, x(t), u(t)) d t$

$\dot{x}=g(t, x(t), u(t))$

$x\left(t_{0}\right)=x_{0}$

and let $\mathrm{u}$ be a control strategy for the differential equation. We assume that $f(t, x(t), u(t))$ and $g(t, x(t), u(t)))$ are continuously differentiable functions in their arguments and concave in the state $\mathrm{x}(\mathrm{t})$ and control $\mathrm{u}(\mathrm{t})$. Suppose $\mathrm{u}^{*}$ and $\mathrm{x}^{*}(\mathrm{t})$ are optimal for the given objective functional and state equation. We define the adjoint variable $\lambda(\mathrm{t})$ as a piecewise differentiable function.

The problem of optimal control is stated as follows

Minimize the number of infected individuals within the time horizon $\mathrm{T}$, that is $\mathrm{t} \in[0, T]$ given by the function

$$
J=\min _{u \in U} \int_{0}^{T} F(t, u, q)+h(t, u) d t
$$

Subject to the state system of equations (5)

and the control constraint

$U=\left\{u_{j}\right.$ given that $u_{j}(t)$ is Lebesgue measurable, $0 \leq u_{j}(t) \leq 1$, for $\left.j=1,2, t \in[0, t]\right\}$

Where $F(t, u, q)=I(t)$

$h(t, u)=\frac{1}{2} A u_{1}{ }^{2}(t)+\frac{1}{2} B u_{2}{ }^{2}(t) . A$ and $B$ are the weights attached for vaccinating susceptible individuals and for the quarantine of infected (symptomatic) individuals respectively. $F(t, u, q)$ defines actual costs while $h(t, u)$ defines the background costs (such as ordering, shipment and distribution, and storage).

Therefore the optimal control problem is to minimize three variables, the infected population, vaccination rate, and quarantine rate. We define the objective functional as

$\min _{u_{1}, u_{2}} J=\min _{u_{1}, u_{2}} \int_{0}^{T}\left[I(t)+\frac{1}{2} A u_{1}^{2}(t)+\frac{1}{2} B u_{2}^{2}(t)\right] d t$

We find optimal control pair $\left(u_{1}^{*}, u_{2}^{*}\right)$ such that

$J\left(u_{1}^{*}, u_{2}^{*}\right)=\operatorname{Min}\left\{J\left(u_{1}, u_{2}\right) ;\left(u_{1}, u_{2}\right) \in U\right\}$ 
Now applying the necessary condition from Pontryagin's Maximum Principle (PMP), we have the problem minimizing a Hamiltonian, $\mathrm{H}$ pointwisely with respect to $u_{1}$ and $u_{2}$

Theorem: Given an optimal control pair $U^{*}(t)=\left(u_{1}^{*}(t), u_{2}^{*}(t)\right)$ and a solution $P^{*}(t)=\left(S^{*}(t), E^{*}(t), I^{*}(t), R^{*}(t)\right)$ of the corresponding state system (5) there exist adjoint variables $\lambda_{S}(t), \lambda_{E}(t), \lambda_{I}(t)$ and $\lambda_{R}(t)$ such that, the Hamiltonian is given by

$$
\begin{aligned}
H(S, E, I, R)= & I(t)+\frac{1}{2} A u_{1}{ }^{2}(t)+\frac{1}{2} B u_{2}{ }^{2}(t)+\lambda_{S}\left(-\frac{\beta S(I+q E)}{N}-u_{1} S\right) \\
& +\lambda_{E}\left(\frac{\beta S(I+q E)}{N}-\delta E\right)+\lambda_{I}\left(\delta E-\left(u_{2}+\gamma\right) I\right) \\
& +\lambda_{R}\left(\gamma I+u_{1} S+u_{2} I\right)
\end{aligned}
$$

Using the Hamiltonian, we derive the adjoint equations. Since the system (5) has four equations, there will be one adjoint equation for each of the S, E, I, and R compartments. The adjoint equation for S is defined by setting the time derivative of the adjoint variable equal to $-\frac{\partial H}{\partial S}$. The other adjoint variables are defined similarly

And $\lambda_{S}^{\prime}=\lambda_{S}\left(\frac{\beta(I+q E)}{N}-u_{1}\right)-\lambda_{E}\left(\frac{\beta(I+q E)}{N}\right)-\lambda_{R} u_{1}$

$\lambda_{E}^{\prime}=\lambda_{S}\left(\frac{\beta S}{N}\right)-\lambda_{E}\left(\frac{\beta S}{N}-\delta\right)-\lambda_{I} \delta$

$\lambda_{I}^{\prime}=-1+\lambda_{S}\left(\frac{\beta S}{N}\right)-\lambda_{E}\left(\frac{\beta S}{N}\right)+\lambda_{I}\left(u_{2}+\gamma\right)-\lambda_{R}\left(\gamma+u_{2}\right) \quad$ and

$\lambda_{R}^{\prime}=0$

We determine the optimal control with optimality conditions

$$
\lambda_{S}(T)=\lambda_{E}(T)=\lambda_{I}(T)=\lambda_{R}(T)=0
$$

Solving for $u_{1}$ and $u_{2}$, we get the optimal control $u_{1}^{*}$ and $u_{2}^{*}$ given by

$$
\begin{aligned}
& u_{1}^{*}=\frac{\left(\lambda_{S}-\lambda_{R}\right) S}{A} \\
& u_{2}^{*}=\frac{\left(\lambda_{I}-\lambda_{R}\right) I}{B}
\end{aligned}
$$

\section{Numerical Illustration}

For illustration purpose we use the estimated values of $\beta=0.195$ (Guinea), 0.217 (Liberia), 0.293 (Sierra Leone), $\delta=0.188$ (Guinea), 0.251 (Liberia), 0.223 (Sierra Leone), $\gamma=0.16$ (Guinea), 0.16 (Liberia), 0.21 (Sierra Leone), $0 \leq u_{1} \leq 1,0 \leq u_{2} \leq 1$ and $0 \leq q \leq 1$. The initial conditions for (a) Guinea: $\mathrm{S}(0)=12500$, $\mathrm{E}(0)=1, \mathrm{I}(0)=12, \mathrm{R}(0)=0$ (b) Liberia: $\mathrm{S}(0)=4800, \mathrm{E}(0)=1, \mathrm{I}(0)=5, \mathrm{R}(0)=0$ and (c) Sierra Leone: $\mathrm{S}(0)=$ $19000, \mathrm{E}(0)=2, \mathrm{I}(0)=25, \mathrm{R}(0)=0$.

Figures 13, 14 and 15 (a,b,c,d) show the effect of vaccination and quarantine on Susceptible, Exposed, Infected, and Recovered compartments, respectively. The blue lines represent no intervention strategy, which is just the disease running its course through the population and eventually dying out. The disease dies out because there is no birth rate (recruitment of susceptible) in the model. The red curves indicate each compartment with an optimal intervention strategy, which reduces the amount of susceptible, exposed, and infected in the population. As a result of the two control measures, vaccination and the quarantine application the recovered population increases. The Figure $13(\mathrm{a}, \mathrm{b}, \mathrm{c}, \mathrm{d})$ for the controls gives us the useful information about possible implementation of intervention strategy for Guinea, The Figure 14 (a,b,c,d) for the controls gives us the useful information about possible implementation of intervention strategy in case Liberia and The Figure 15 (a,b,c,d) for the controls give us the useful information about possible implementation of intervention strategy for Sierra Leone .

\section{Conclusion}

Vaccination and quarantine are two controls that help to contain the spread of the disease. Although there is currently no vaccine available for ebola disease, since no cost is available, we use the model with arbitrary price values for the vaccine. This will allow us to make predictions about how a vaccine should be implemented once it is readily available for use. When infected people are quarantined, the number of contacts that can transmit the disease automatically decreases. Therefore application of quarantine even in the absence of vaccination plays a very important rule in combating against disease. 


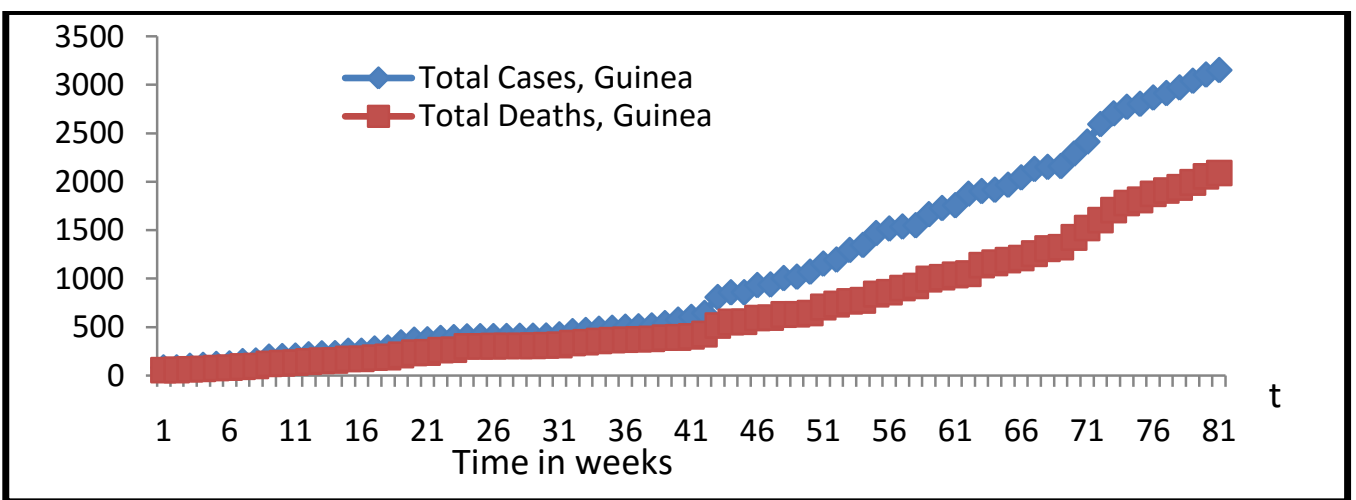

Figure 3: Cumulative number of infected cases and deaths due to 2014 Ebola outbreak for Guinea

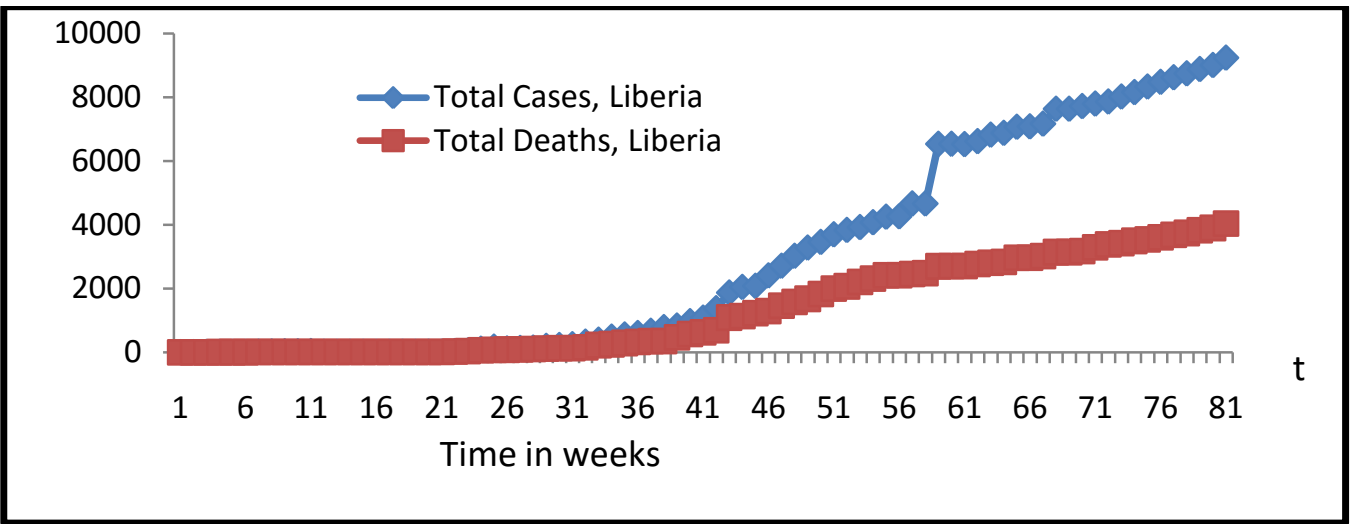

Figure 4: Cumulative number of infected cases and deaths due to 2014 Ebola outbreak for Liberia

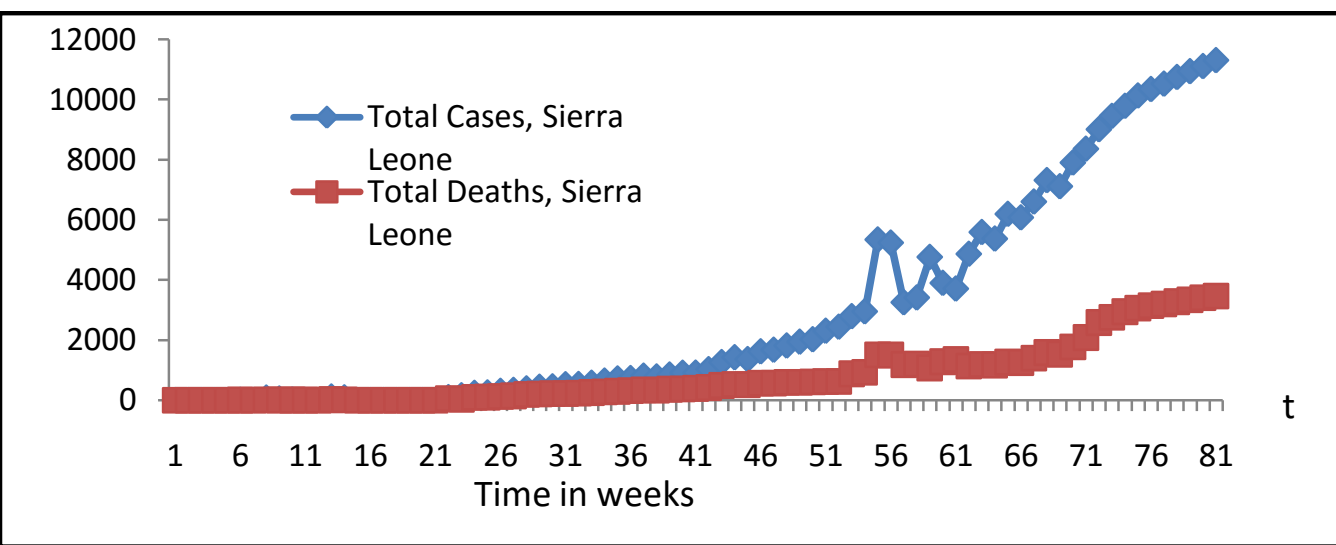

Figure 5: Cumulative number of infected cases and deaths due to 2014 Ebola outbreak for Sierra Leone

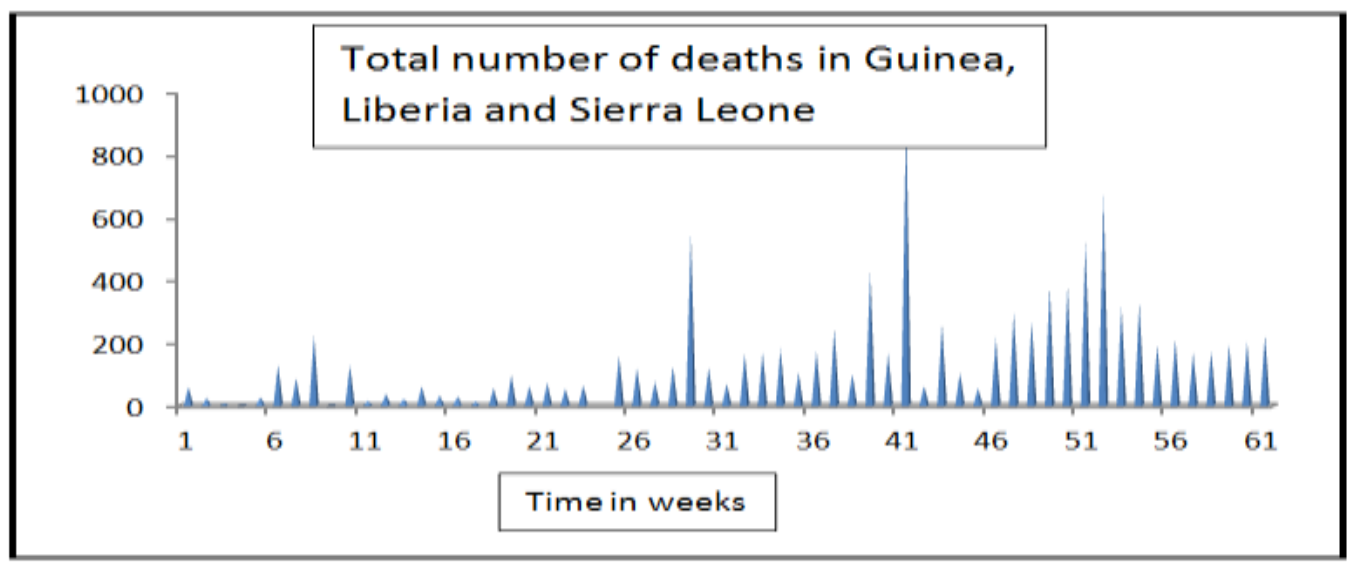

Figure 6: Total number of deaths due Ebola outbreak in West Africa 


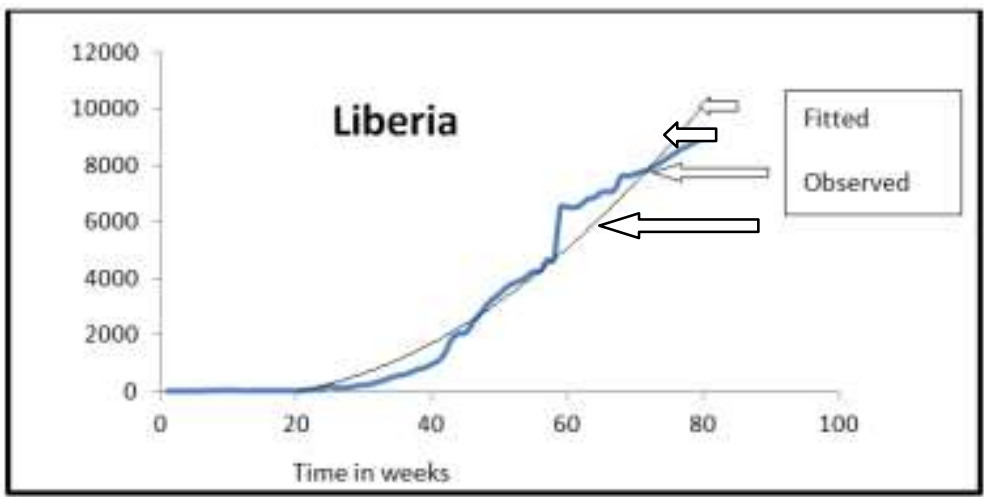

Figure 7: Cumulative number of infected with Ebola outbreak and fitted values for Liberia

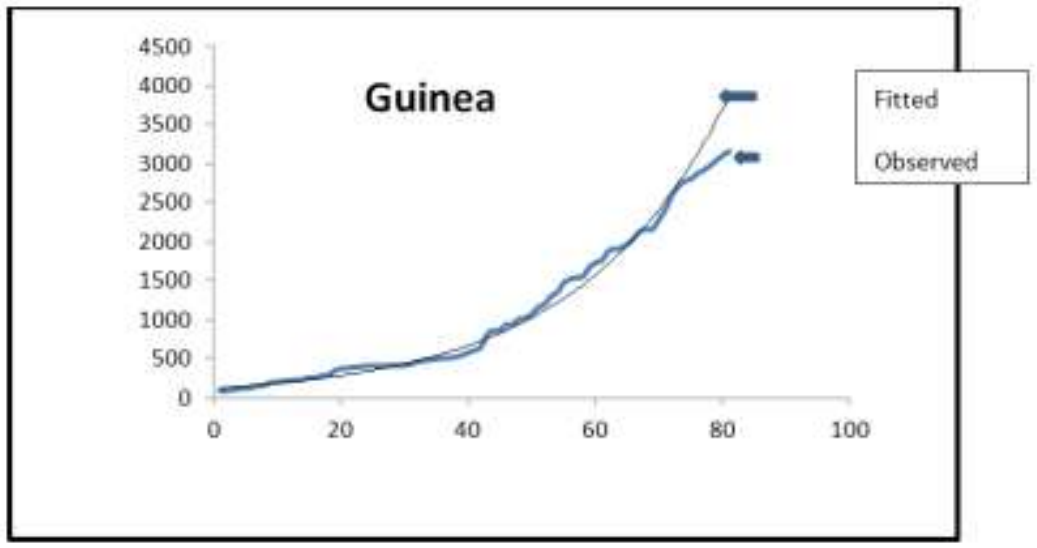

Figure 8: Cumulative number of infected with Ebola outbreak and fitted values for Guinea

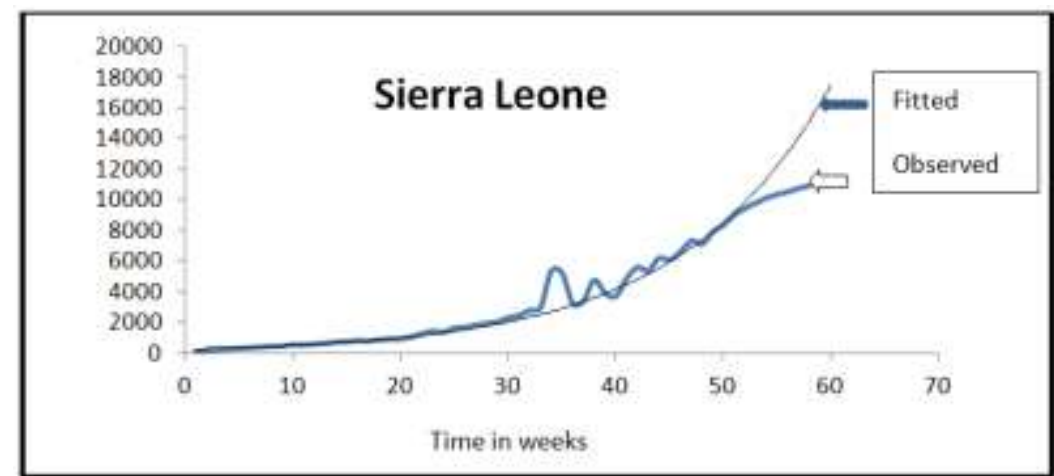

Figure 9: Cumulative number of infected with Ebola outbreak and fitted values for Sierra Lione

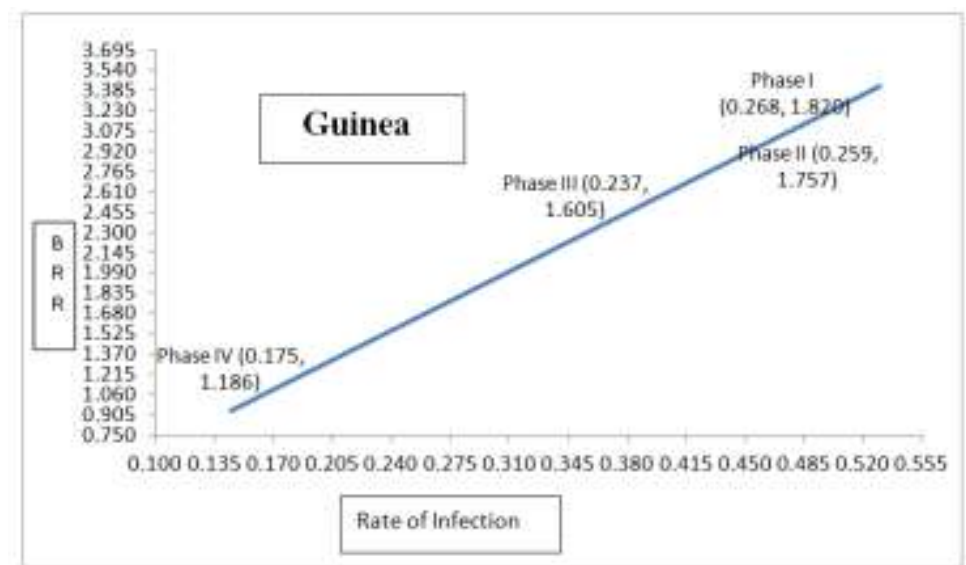

Figure 10: Estimation of $\beta$ and $R_{0}$ at various exponential growth rates using ebola outbreak data of Guinea. 


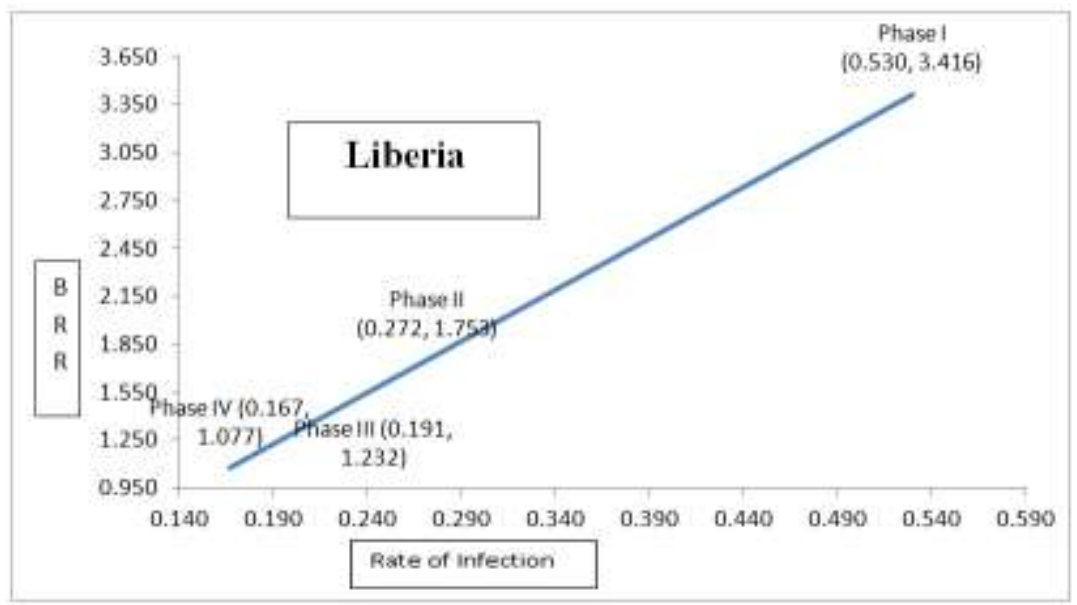

Figure 11: Estimation of $\beta$ and $R_{0}$ at various exponential growth rates using ebola outbreak data of Liberia.

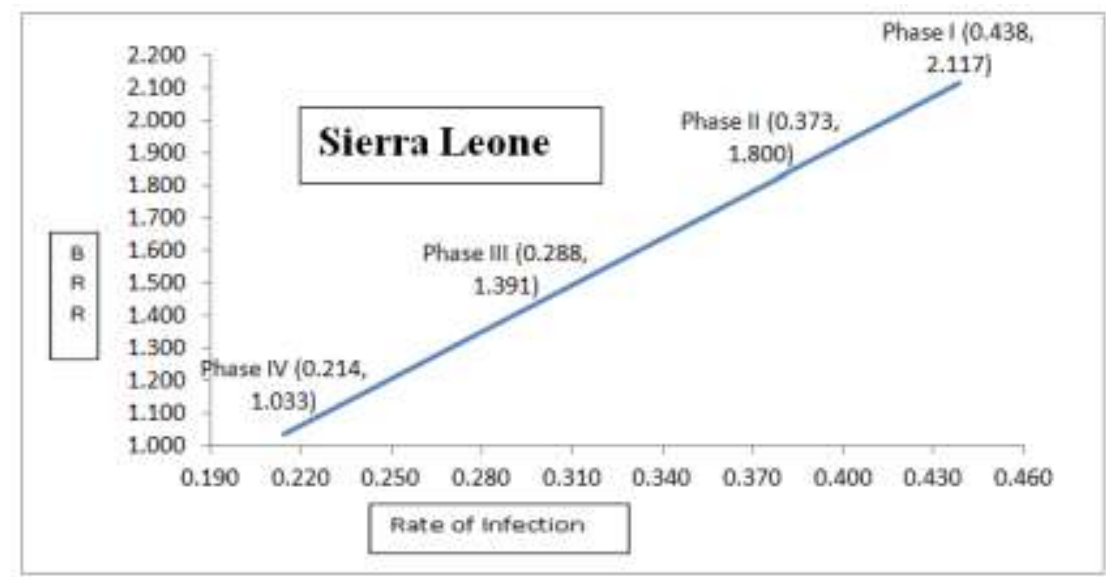

Figure 12: Estimation of $\beta$ and $R_{0}$ at various exponential growth rates using ebola outbreak data of Seirra Leone.

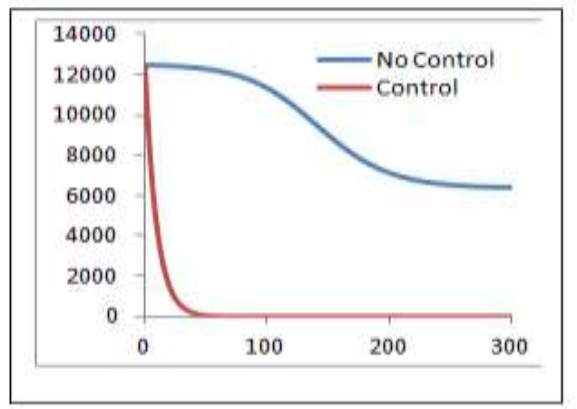

(a)

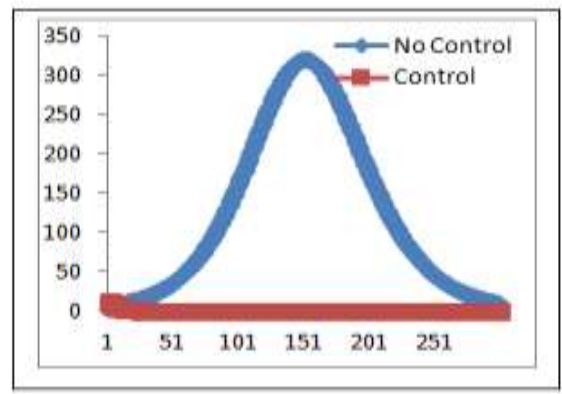

(c)

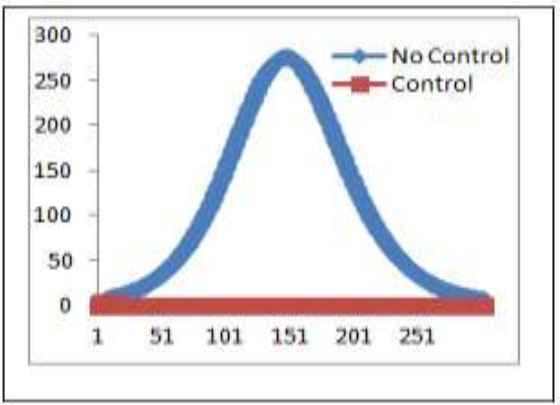

(b)

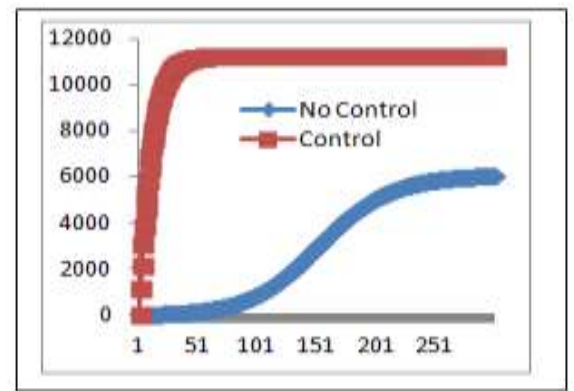

(d)

Figure 13: Effect of vaccination and quarantine (with control) and without control on susceptible (a), exposed (b), infected (c) and recovered (d) individuals for Guinea. 


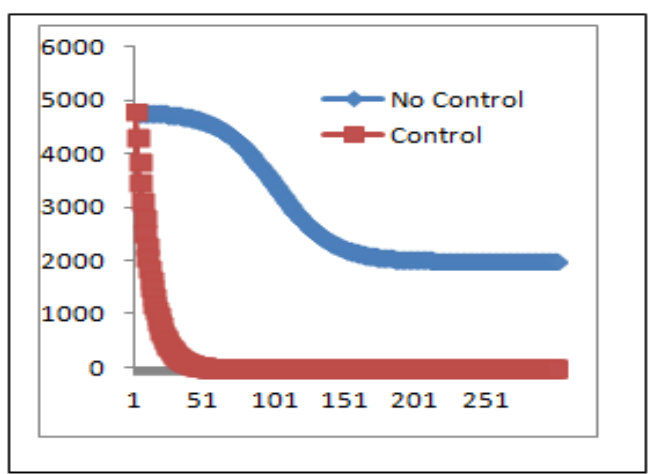

(a)

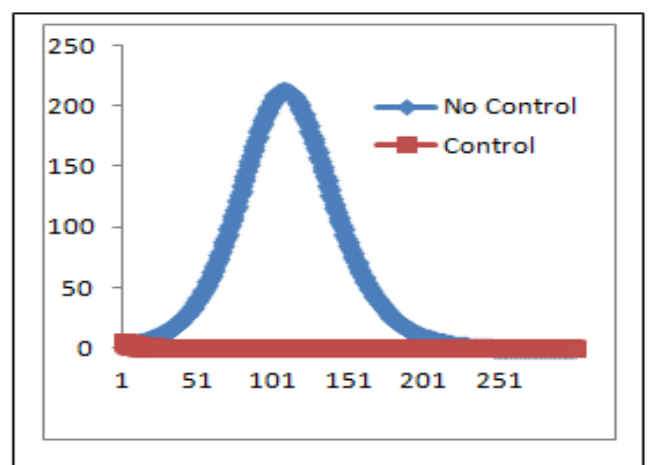

(c)

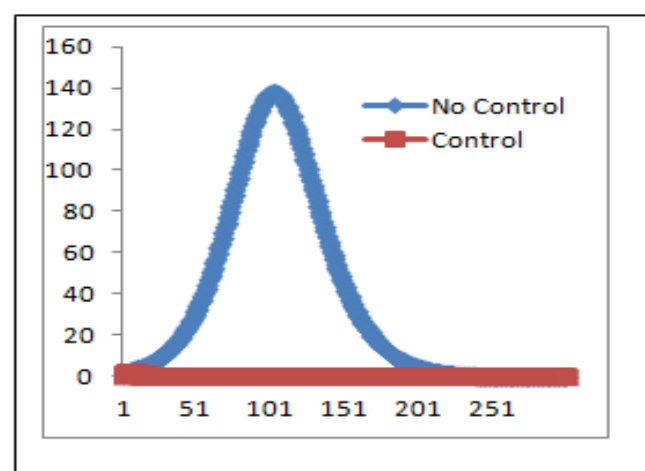

(b)

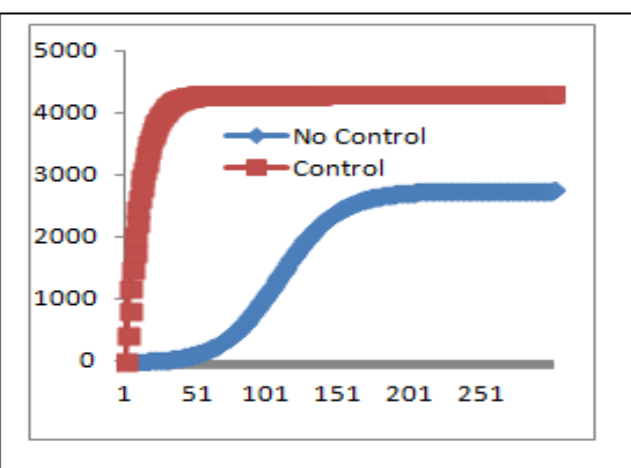

(d)

Figure 14: Effect of vaccination and quarantine (with control) and without control on susceptible (a), exposed (b), infected (c) and recovered (d) individuals for Liberia.

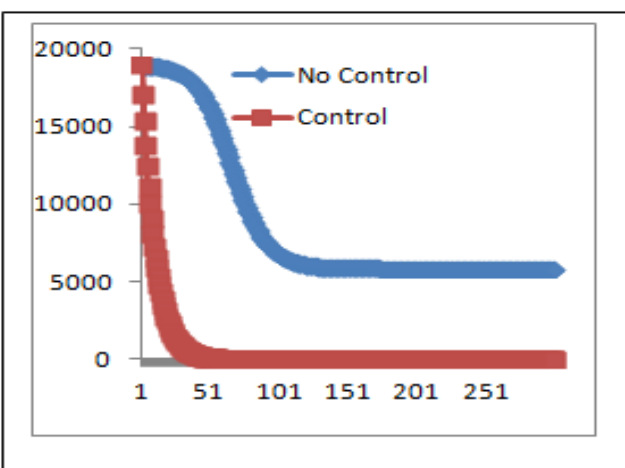

(a)

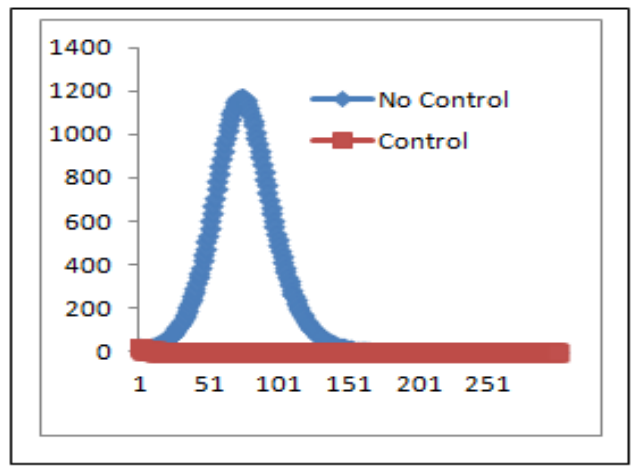

(c)

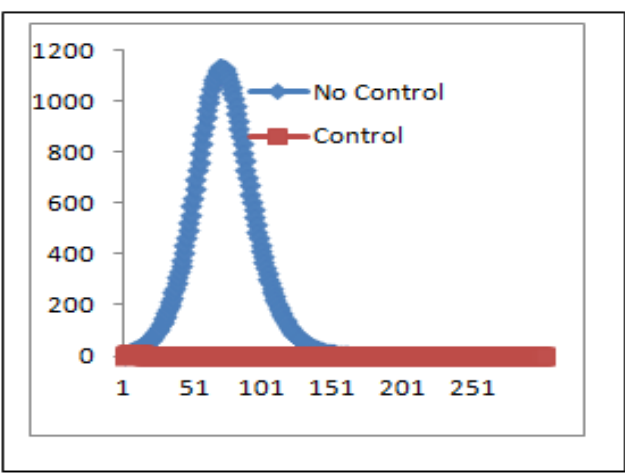

(b)

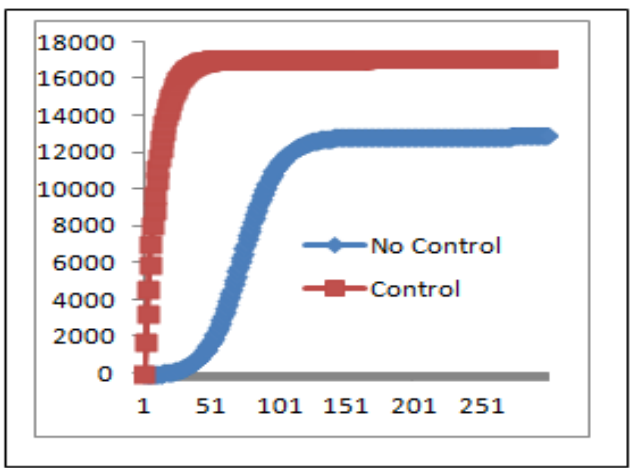

(d)

Figure 15: Effect of vaccination and quarantine (with control) and without control on susceptible (a), exposed (b), infected (c) and recovered (d) individuals for Sierra Leone. 


\section{References}

[1]. Adapted by CRS from the Centers of Disease Control and Prevention (CDC) at http://www.cdc.gov/vhf/ebola/resources/distribution-map-guinea-outbreak.html

[2]. Astacio, J., Briere, D., Guillen, M., Martinez, J., Rodriguez, F. and Valenzuela-Campos, N. Mathematical models to study the outbreaks of Ebola. Research Report, 1996.

[3]. Breman, J.G., Piot, P., Johnson, K.M., et al., The epidemiology of Ebola hemorrhagic fever in Zaire, 1976.Proceedings of the International Colloquium on Ebola Virus Infections held in Antwerp, Belgium, 6-8 December, 1977.

[4]. Chowell, G., Hengartnerb, N.W., Castillo-Chavez, C., Fenimorea, P.W. and Hymanc, J.M. (2005a). The basic reproductive number of Ebola and the effects of public health measures: the cases of Congo and Uganda, Journal of Theoretical Biology

[5]. Chowell, G., Castillo-Chavez, C., Fenimore, P.W., et al., (2004b). Implications of an uncertainty and sensitivity analysis for SARS's basic reproductive number for general public health measures, Emerging Infectious Diseases.

[6]. Created by CRS from WHO, Ebola Virus Disease, fact sheet, number 103, April 2014 and WHO, Ebola Virus Disease Update-West Africa, August 22, 2014.

[7]. https://www.cdc.gov/vhf/ebola/outbreaks/2014-west-africa/

[8]. https://www.cdc.gov/vhf/ebola/outbreaks/2014-west-africa/previous-updates.html

[9]. Lekone, Phenyo E. and Finkenstädt, Bärbel F. (2006). Statistical Inference in a Stochastic Epidemic SEIR Model with Control Intervention: Ebola as a Case Study, Biometrics 62, 1170-1177.

[10]. Li, Z., Teng, Z., Feng, X., Li, Y. and Zhang, H. (2015). Dynamical Analysis of an SEIT Epidemic Model with Application to Ebola Virus Transmission in Guinea, Computational and Mathematical Methods in Medicine ,Hindawi Publishing Corporation, Article ID 582625, 11 pages

[11]. Lenhart, S. and Workman, J. Optimal control applied to biological models, CRC Press, 2007.

[12]. Piot, P., Sureau, P., Breman, J.G, Heymann, D.L., et al. Clinical Aspects of Ebola Virus Infection in Yambuku Area, Zaire, 1976: Proceedings of an International Colloquium on Ebola Virus Infection and Other Haemorrhagic Fevers held in Antwerp, Belgium, 6-8 December, 1977. 